\author{
R. J. MacIsaac $\cdot$ C. Tsalamandris $\cdot$ M. C. Thomas • \\ E. Premaratne $\cdot$ S. Panagiotopoulos $\cdot$ T. J. Smith $\cdot$ \\ A. Poon - M. A. Jenkins · S. I. Ratnaike - D. A. Power • \\ G. Jerums
}

\title{
Estimating glomerular filtration rate in diabetes: a comparison of cystatin-C- and creatinine-based methods
}

Received: 13 January 2006 / Accepted: 14 March 2006 / Published online: 3 May 2006

C) Springer-Verlag 2006

\begin{abstract}
Aims/hypothesis: We compared the predictive performance of a GFR based on serum cystatin C levels with commonly used creatinine-based methods in subjects with diabetes. Subjects, materials and methods: In a crosssectional study of 251 consecutive clinic patients, the mean reference (plasma clearance of ${ }^{99 \mathrm{~m}} \mathrm{Tc}$-diethylene-triaminepenta-acetic acid) GFR (iGFR) was $88 \pm 2 \mathrm{ml} \mathrm{min}^{-1} 1.73 \mathrm{~m}^{-2}$. A regression equation describing the relationship between iGFR and 1/cystatin $\mathrm{C}$ levels was derived from a test population $(n=125)$ to allow for the estimation of GFR by cystatin C (eGFR-cystatin C). The predictive performance of eGFR-cystatin C, the Modification of Diet in Renal Disease 4 variable formula (MDRD-4) and Cockcroft-Gault (C-G)
\end{abstract}

R. J. MacIsaac $(\bowtie) \cdot$ C. Tsalamandris · E. Premaratne

S. Panagiotopoulos · T. J. Smith · G. Jerums

Endocrine Centre and the Department of Medicine,

Austin Health and University of Melbourne,

Heidelberg Repatriation Hospital,

Level 2, Centaur Building,

Heidelberg West, VIC, 3081, Australia

e-mail: r.macisaac@unimelb.edu.au

Tel.: +61-3-94965489

Fax: +61-3-94963365

M. C. Thomas

Danielle Alberti Memorial Centre for Diabetes Complications,

Baker Medical Research Institute,

Melbourne, VIC, Australia

\section{A. Poon}

Division of Nuclear Medicine and Centre for PET,

Austin Health,

Heidelberg, VIC, Australia

M. A. Jenkins · S. I. Ratnaike

Division of Laboratory Medicine, Austin Health,

Heidelberg, VIC, Australia

D. A. Power

Department of Nephrology, Austin Health,

Heidelberg, VIC, Australia formulas were then compared in a validation population $(n=126)$. Results: There was no difference in renal function $(\mathrm{ml} \mathrm{min})^{-1} 1.73 \mathrm{~m}^{-2}$ ) as measured by iGFR (89.2 \pm 3.0$)$, eGFR-cystatin C $(86.8 \pm 2.5)$, MDRD-4 $(87.0 \pm 2.8)$ or $\mathrm{C}-\mathrm{G}$ (92.3 \pm 3.5$)$. All three estimates of renal function had similar precision and accuracy. Conclusions/interpretation: Estimates of GFR based solely on serum cystatin C levels had the same predictive potential when compared with the MDRD -4 and $\mathrm{C}-\mathrm{G}$ formulas.

Keywords Cockcroft-Gault - Cystatin C - Diabetic nephropathy · Glomerular filtration rate $\cdot$ MDRD . Microalbuminuria $\cdot$ Renal insufficiency

Abbreviations $\mathrm{C}-\mathrm{G}$ formula: Cockcroft-Gault formula . eGFR-cystatin C: estimation of GFR by cystatin C - iGFR: reference GFR (plasma clearance of ${ }^{99 \mathrm{~m}} \mathrm{Tc}$-diethylenetriamine-penta-acetic acid) - MDRD-4 formula: modification of diet in renal disease 4 variable formula

\section{Introduction}

The measurement of serum cystatin $\mathrm{C}$, a low molecular weight protein $(13 \mathrm{kDa})$ that is freely filtered through the glomerulus and almost completely reabsorbed and catabolised by tubular cells, has been proposed as a simple, reliable and accurate marker of GFR [1]. It has recently been shown that GFR derived from a simple regression equation based on the relationship between the reciprocal of serum cystatin $\mathrm{C}$ levels and GFR measured by ${ }^{125} \mathrm{I}$ iothalamate clearance was a more accurate estimate of renal function than the Cockcroft-Gault $(\mathrm{C}-\mathrm{G})$ formulas in subjects with and without diabetes [2]. However, the bias, precision (standard deviation of the difference from a reference method) and accuracy (within 15, 30 and $50 \%$ of reference method values) for a GFR estimated from serum cystatin C levels have not been compared with the abbreviated Modification of Diet in Renal Disease 4 variable formula (MDRD-4) or $\mathrm{C}-\mathrm{G}$ formula in patients with diabetes and a wide range of renal function. 


\section{Subjects, materials and methods}

Patient population and study design

This study was a cross-sectional survey of patients attending the diabetes clinic at Austin Health, a tertiary referral centre and teaching hospital of the University of Melbourne, Victoria, Australia. The study population consisted of 251 consecutive patients who had a mean reference GFR (plasma clearance of ${ }^{99 \mathrm{~m}} \mathrm{Tc}$-diethylenetriamine-penta-acetic acid) (iGFR) measurement and sufficient clinical and biochemical data for inclusion. The majority of patients $(92 \%)$ were Caucasian; there were no patients of African-American origin. Patients with known non-diabetic renal disease were excluded. Creatinine and cystatin $\mathrm{C}$ concentrations were measured in a fasting blood sample collected on the morning of the iGFR estimation.

We randomly selected 125 subjects (the test population), from the entire study population and a regression equation describing the relationship between iGFR and the reciprocal of serum cystatin $\mathrm{C}$ levels was derived to allow the estimation of GFR by cystatin C (eGFR-cystatin C). In the remaining 126 subjects (the validation population), the bias, precision and accuracy of eGFR-cystatin $\mathrm{C}$ and both the MDRD-4 and $\mathrm{C}-\mathrm{G}$ formulas were compared. Informed consent was obtained from patients, as approved by the Austin Health Human Research Ethics Committee, for the unrestricted use of clinical data for non-interventional research studies.

\section{Laboratory methods}

The reference value iGFR was obtained by measuring the plasma disappearance of ${ }^{99 \mathrm{~m}} \mathrm{Tc}$-diethylene-triamine-pentaacetic acid employing the Brochner-Mortensen correction [3]. Urinary albumin and serum electrolytes were measured as described previously [3]. Creatinine was measured on an automatic analyser (Hitachi 911; Roche Diagnostics, Mannheim, Germany), which produces creatinine values that fall within less than $\pm 15 \%$ of the reference MDRD method. This method is considered acceptable with respect to bias and precision by the Australian working group on the automatic reporting of estimated GFR [4], and hence we did not adjust serum creatinine concentrations. Cystatin $\mathrm{C}$ was measured using a particle-enhancing immunonephelometric
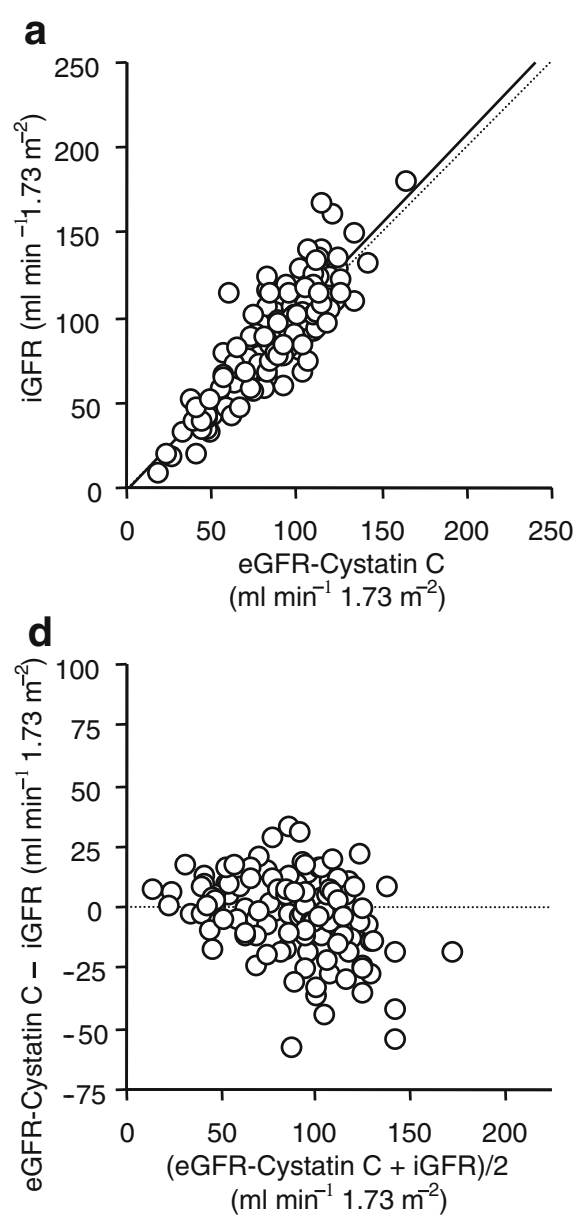
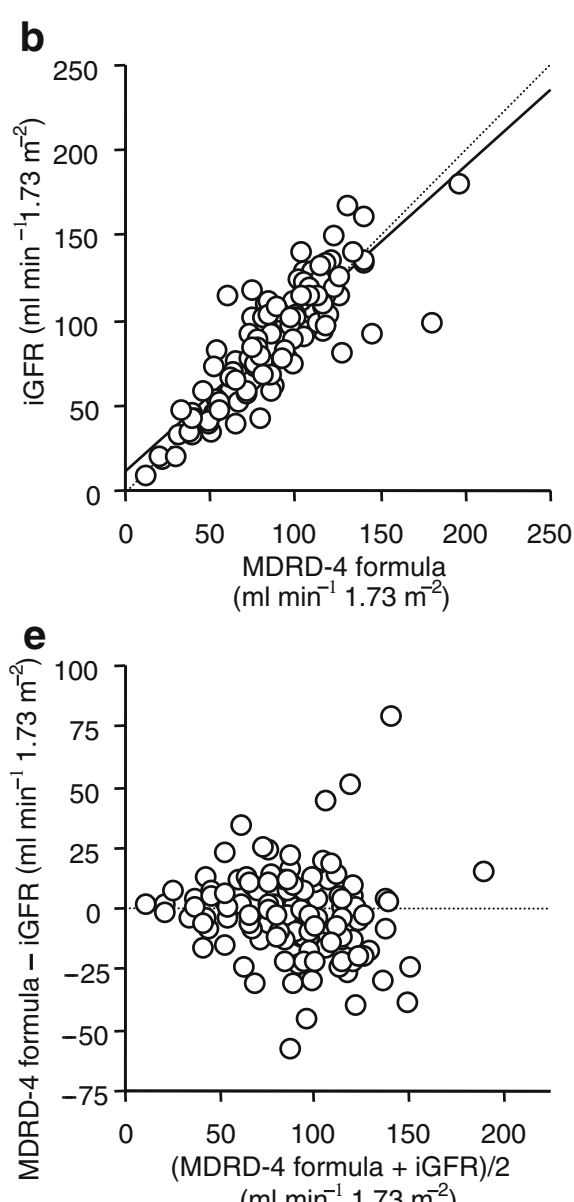

$\left(\mathrm{ml} \mathrm{min}-1.73 \mathrm{~m}^{-2}\right)$
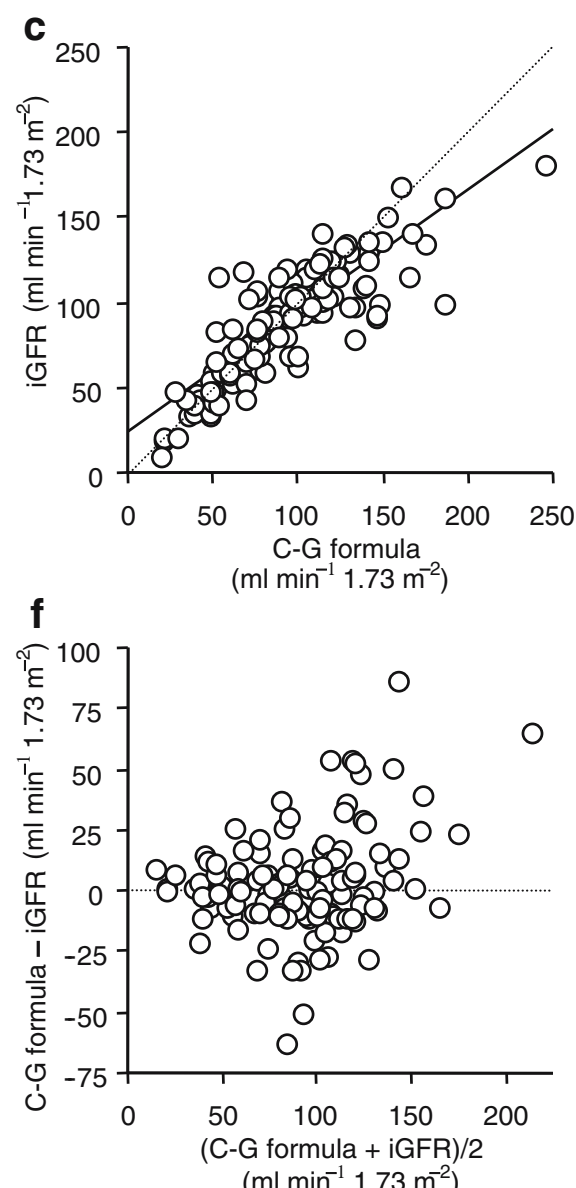

Fig. 1 Comparison of estimates of renal function with reference iGFR (plasma clearance of ${ }^{99 \mathrm{~m}} \mathrm{Tc}$-diethylene-triamine-penta-acetic acid) values for 126 subjects in the validation group. Regression lines for iGFR and eGFR-cystatin C (a), iGFR and the MDRD-4 variable formula (b) and iGFR and the Cockcroft-Gault $(\mathrm{C}-\mathrm{G})$ formula (c). Dotted line, line of identity. The Bland-Altman plot of disagreement between eGFR-cystatin C (d), the MDRD-4 formula (e) and the $\mathrm{C}-\mathrm{G}$ formula (f) with reference iGFR values 
assay (Dade Behring, Marburg, Germany) as described previously [5].

\section{Statistical analysis}

The predictive performance of eGFR-cystatin $\mathrm{C}$, the MDRD-4 and $\mathrm{C}-\mathrm{G}$ formulas was assessed using the methods of Bland-Altman and Manjunath et al. [6]. Accuracy was also estimated within 15,30 and $50 \%$ of iGFR values. The accuracy of each method for estimating renal function was compared by means of chi square tests. For continuous variables, data are means \pm SEM, except where presented as medians (interquartile range). A $p$ value of $<0.05$ was considered statistically significant, and a $95 \%$ level of confidence was used.

\section{Results}

Patient characteristics, correlations with cystatin C and derivation of eGFR-cystatin $\mathrm{C}$

The study population had a mean age of $60 \pm 1$ years (range 22-84) and was comprised mainly of patients with type 2 diabetes $(82 \%)$. The mean measured iGFR was $88 \pm 2 \mathrm{ml}$ $\min ^{-1} 1.73 \mathrm{~m}^{-2}$ (range $9-181$ ) and $22 \%$ of subjects had an iGFR $<60 \mathrm{ml} \mathrm{min}-1.73 \mathrm{~m}^{-2}$. The mean (interquartile range) of serum cystatin $\mathrm{C}$ and creatinine levels was 0.91 $(0.78,1.15)$, range $0.51-416 \mathrm{mg} / \mathrm{l}$, and $76(65,94)$, range 34-354 $\mu \mathrm{mol} / \mathrm{l}$, respectively. The AER status for the study population was 61,25 and $14 \%$ for normo-, micro- and macroalbuminuria respectively. There were no significant differences in the clinical or biochemical characteristics for the entire, test and validation study populations. The regression equation, $\mathrm{GFR}=(84.6 /$ cystatin $\mathrm{C})-3.2(r=0.82$, $p<0.0001)$ was derived from the relationship of iGFR values and the reciprocal of cystatin $\mathrm{C}$ levels in the test population. This equation had the same characteristics as the regression equation derived from the entire study population, i.e. $\mathrm{GFR}=(86.7 /$ cystatin $\mathrm{C})-4.2 \quad(r=0.86$, $p<0.0001)$.

Comparison of eGFR-cystatin and the MDRD-4 and $\mathrm{C}-\mathrm{G}$ formulas

The comparison of the correlations between iGFR values and eGFR-cystatin $\mathrm{C}$, MDRD-4 and the $\mathrm{C}-\mathrm{G}$ formulas, together with their Bland-Altman plots for the validation group are shown in Fig. 1.

The detailed performance of renal function estimated by eGFR-cystatin $\mathrm{C}$ or by the MDRD- 4 and $\mathrm{C}-\mathrm{G}$ formulas for predicting iGFR levels is shown in Table 1 . There was no significant difference in renal function $\left(\mathrm{ml} \mathrm{min}^{-1} 1.7 \mathrm{~m}^{-2}\right)$ as measured by iGFR $(89.2 \pm 3.0)$, eGFR-cystatin C $(86.8 \pm$ $2.5)$ or by the MDRD-4 (87.0 \pm 2.8$)$ and $C-G(92.3 \pm 3.5)$ formulas. Overall, eGFR-cystatin C and the MDRD-4 and $\mathrm{C}-\mathrm{G}$ formulas had similar precision, combined root mean squared error values and accuracy (within 15 and 30\%) when compared to iGFR levels. However, eGFR-cystatin C had a greater accuracy within $50 \%$ of iGFR values than the $C-G$ formula $(\mathrm{p}<0.04)$.

Table 1 Predictive performance of eGFR-cystatin C, the MDRD-4 variable (MDRD-4) formula and Cockcroft-Gault (C-G) formula in the validation population $(n=126)$

\begin{tabular}{llll}
\hline Equation & eGFR-cystatin C $(n=126)$ & MDRD-4 $(n=126)$ & $\mathrm{C}-\mathrm{G}(n=126)$ \\
\hline Gradient $(95 \% \mathrm{CI})$ & $1.04(0.94$ to 1.15$)$ & $0.90(0.79$ to 1.00$)$ & $0.71(0.63$ to 0.79$)$ \\
Intercept $(95 \% \mathrm{CI})$ & $-1.5(-11.1$ to 8.1$)$ & $11.3(1.8$ to 20.8$)$ & $23.5(15.3$ to 31.7$)$ \\
$R$ & 0.87 & 0.84 & 0.84 \\
$r^{2}$ & 0.75 & 0.70 & 0.70 \\
$p$ & $<0.0001$ & $<0.0001$ & $<0.0001$ \\
eGFR-cystatin/MDRD-4/ C-G (ml min $\left.{ }^{-1} 1.73 \mathrm{~m}^{-2}\right)$ & $86.8 \pm 2.5$ & $87.0 \pm 2.8$ & $92.3 \pm 3.5$ \\
eGFR-cystatin/MDRD-4/ C-G (95\% CI) & 82.0 to 91.7 & 81.5 to 92.4 & 85.4 to 99.2 \\
Bias & -2.4 & -2.2 & 3.1 \\
Precision & 16.4 & 18.3 & 21.2 \\
CRMSE & 16.6 & 18.4 & 21.4 \\
Accuracy within 15\% & $59 \%$ & $60 \%$ & $63 \%$ \\
Accuracy within 30\% & $88 \%$ & $89 \%$ & $81 \%$ \\
Accuracy within 50\% & $98 \%$ & $96 \%$ & $94 \%{ }^{\mathrm{a}}$ \\
\hline
\end{tabular}

The mean iGFR (plasma clearance of ${ }^{99 \mathrm{~m}}$ Tc-diethylene-triamine-penta-acetic acid) level for the validation group was $89.2 \pm 3.0$ $\left(\mathrm{ml} \mathrm{min} \min ^{-1} 1.73 \mathrm{~m}^{-2}\right)$

Bias: the mean difference between each marker of renal function and iGFR; Precision: the standard deviation of the above difference; CRMSE: combined root mean squared error, calculated as the square root of the [(mean difference in estimate- observed $)^{2}+$ (standard deviation of the difference) $\left.{ }^{2}\right]$; $e G F R$ : estimated GFR based on unadjusted serum cystatin C levels; $M D R D-4$ : the simplified four variable MDRD formula; $C-G$ : Cockcroft-Gault formula

${ }^{a} p<0.04$ vs eGFR-cystatin C 


\section{Discussion}

Using established methods to develop and evaluate prediction equations, a GFR estimated solely from serum cystatin $\mathrm{C}$ levels had a predictive performance equal to commonly used creatinine-based estimates of renal function in a population of diabetic subjects with a wide range of renal function. In particular, bias, precision and accuracy of the different prediction equations were similar, although eGFRcystatin $\mathrm{C}$ had a slightly greater accuracy within $50 \%$ of reference iGFR values than the $\mathrm{C}-\mathrm{G}$ formula. It is doubtful whether this difference has any clinical significance.

In agreement with previous studies, we found that the MDRD-4 equations slightly underestimated $(-2.2 \mathrm{ml}$ $\min ^{-1} 1.73 \mathrm{~mm}^{-2}$ ), whereas the $\mathrm{C}-\mathrm{G}$ formula slightly overestimated $\left(+3.1 \mathrm{ml} \mathrm{min}^{-1} 1.73 \mathrm{~m}^{-2}\right)$ reference iGFR measurements. Also, like others, we found that a calculated GFR largely lacked accuracy and precision for estimating a reference isotopic GFR method [7]. Only two-thirds of values estimated by the three methods used fell within $15 \%$ of the reference iGFR measurements.

Many studies have demonstrated the superiority of serum cystatin $\mathrm{C}$ compared with serum creatinine measurements as a marker of renal function in subjects with diabetes, although this has not been a universal finding. Fewer studies have compared serum cystatin $\mathrm{C}$ levels and the $\mathrm{C}-\mathrm{G}$ formula and, to our knowledge, this study is the first to incorporate a comparison of eGFR-cystatin $\mathrm{C}$ with both the MDRD-4 and C-G formulas. Recently, trends in the reciprocal of cystatin $C$ serum levels over a 4-year period were demonstrated to correlate more closely with iothalamate clearance than the MDRD-4 and $\mathrm{C}-\mathrm{G}$ formulas in subjects with type 2 diabetes and an initial GFR $>120 \mathrm{ml}$ $\min ^{-1} 1.73 \mathrm{~m}^{-2}$ [8]. The possible superiority of serum cystatin $\mathrm{C}$ compared with serum creatinine for predicting GFR may be explained by the greater influence of factors, apart from renal clearance, on creatinine production and secretion such as renal tubular secretion, age, sex and muscle mass.

Although multiple factors apart from renal function may influence serum cystatin C levels [9], we found that GFR calculated solely from serum cystatin $\mathrm{C}$ levels and not adjusted for clinical or biochemical variables compared favourably with creatinine-based methods that account for some of the factors known to influence creatinine production. In healthy subjects, cystatin $\mathrm{C}$ levels have been reported to increase with age, and age-specific reference ranges have been proposed [9]. Possibly, an adjustment for age would lead to an even better predictive performance of an eGFR-cystatin formula, as happens when effect of age is incorporated in creatinine-based formulas used to estimate renal function.

The measurement of serum cystatin $\mathrm{C}$ levels in subjects with diabetes may have practical implications apart from providing an estimation of renal function. Thus there is also evidence to suggest that serum cystatin $\mathrm{C}$ levels predict cardiovascular risk better than serum creatinine levels or GFR levels estimated by the MDRD formula [10]. A rigorous cost-effectiveness analysis for measuring cystatin
C levels as opposed to measuring creatinine levels and subsequently estimating a GFR using established formulas has not been performed to date. We estimate that a single cystatin $\mathrm{C}$ measurement, using the method employed in this study, would cost approximately A \$6- (Australian dollars). In comparison, measurement of a single creatinine level on the auto analyser used in this study (Hitachi 911) is estimated to cost only A $\$ 1.75$. The current lack of an automated method for measuring cystatin C levels undoubtedly accounts for a significant proportion of the increased cost associated with measuring cystatin C levels.

It is not our intention that the formula used in this study should be applied in clinical practice as a means of estimating GFR from serum cystatin C levels. However, we do hope that our findings, which also support the literature describing the potential usefulness of cystatin $\mathrm{C}$ as a marker of GFR, will trigger large population studies resulting in a further validation and possible improvement of the predictive performance of an estimated GFR based on measurement of serum cystatin $\mathrm{C}$ levels for subjects with and without diabetes.

Acknowledgement This work was supported by the National Health and Medical Research Council of Australia (Grant number 266505).

\section{References}

1. Filler G, Bokenkamp A, Hofmann W, Le Bricon T, MartinezBru C, Grubb A (2005) Cystatin C as a marker of GFR history, indications, and future research. Clin Biochem 38:1-8

2. Hoek FJ, Kemperman FA, Krediet RT (2003) A comparison between cystatin $\mathrm{C}$, plasma creatinine and the Cockcroft and Gault formula for the estimation of glomerular filtration rate. Nephrol Dial Transplant 18:2024-2031

3. Premaratne E, MacIsaac RJ, Tsalamandris C, Panagiotopoulos S, Smith T, Jerums G (2005) Renal hyperfiltration in type 2 diabetes: effect of age related decline in GFR. Diabetologia 48:2486-2493

4. Mathew T on behalf of the Australasian Creatinine Consensus Working Group (2005) Chronic kidney disease and automatic reporting of estimated glomerular filtration rate: a position statement. Med J Aust 183:138-141

5. Jenkins MA, Brown DJ, Ierino FL, Ratnaike SI (2003) Cystatin $\mathrm{C}$ for estimation of glomerular filtration rate in patients with spinal cord injury. Ann Clin Biochem 40:364-368

6. Manjunath G, Sarnak MJ, Levey AS (2001) Prediction equations to estimate glomerular filtration rate: an update. Curr Opin Nephrol Hypertens 10:785-792

7. Poggio ED, Wang X, Greene T, Van Lente F, Hall PM (2005) Performance of the modification of diet in renal disease and Cockcroft-Gault equations in the estimation of GFR in health and in chronic kidney disease. J Am Soc Nephrol 16:459-466

8. Perkins BA, Nelson RG, Ostrander BE et al (2005) Detection of renal function decline in patients with diabetes and normal or elevated GFR by serial measurements of serum cystatin C concentration: results of a 4-year follow-up study. J Am Soc Nephrol 16:1404-1412

9. Wasen E, Isoaho R, Mattila K, Vahlberg T, Kivela SL, Irjala K (2003) Serum cystatin C in the aged: relationships with health status. Am J Kidney Dis 42:36-43

10. Shlipak MG, Sarnak MJ, Katz R et al (2005) Cystatin C and the risk of death and cardiovascular events among elderly persons. N Engl J Med 352:2049-2060 